\title{
On multivariate discounted compound renewal sums with time-dependent claims in the presence of reporting/payment delays
}

\author{
Jae-Kyung Woo*
}

September 5, 2016

\begin{abstract}
In this paper, we consider an insurance portfolio containing several types of policies which may simultaneously face claims arising from the same catastrophe. A renewal counting process for the number of events causing claims and multivariate claim severities which are dependent on the occurrence time and/or the delay in reporting or payment are assumed. A unified model is proposed to study the time-dependent loss quantities such as the discounted aggregate reported/unreported claims and the number of the incurred but not reported (IBNR) claims. We then derive the joint moments of (i) different types of discounted aggregate claims until time $t$; and (ii) different types of discounted aggregate reported/unreported claims (including the total numbers of IBNR as special case) until time $t$. Finally, some numerical examples involving covariances and correlations of the aforementioned quantities are provided.
\end{abstract}

Keywords: Multiline insurance; Renewal process; Multivariate distribution; Discounted aggregate claim costs; Reported/Unreported claims; IBNR claims; Joint moments; Covariance; Correlation.

\section{Introduction}

Aggregation of dependent risks in multiline insurance has attracted considerable attention in the actuarial industry. For example, different types of damages of property or casualty could be induced by the same catastrophic event such as flooding or earthquake in non-life insurance, whereas the deaths of a couple can be the result of a common accident in life insurance. Risk modeling in such cases requires the construction of multivariate probability distributions (e.g. multivariate normal distribution by Panjer (2002), multivariate Tweedie distribution by Furman and Landsman (2010), and multivariate Pareto distribution by Chiragiev and Landsman (2007) and Asimit et al. (2010)) or copulas (e.g. Joe (1997), Archimedean copula in Alink et al. (2005), Farlie-Gumbel-Morgenstern copula with mixed Erlang marginals in Cossette et al. (2013)). Similar to the class of multivariate phase-type (MPH) distributions, which has a number of nice properties and applications (e.g. Assaf et al. (1984), Kulkarni (1989), Cai and Li (2005a,b)), there are various useful results for the class of multivariate Erlang mixtures (e.g. Lee and Lin (2012), Willmot and Woo (2015)).

However, modeling aggregate losses of multiline (re)insurance in the presence of dependencies not only across policies or business lines but also on their incurral times is more complicated and challenging. It requires special attention to adequately reflect the association between stochastic assumption on the number of events causing losses and multivariate assumption on the claim amounts. For one

*Department of Statistics and Actuarial Science, University of Hong Kong, Pokfulam Road, Hong Kong. Email address: jkwoo@hku.hk 
dimensional case, a wide variety of stochastic models have been considered. In particular, a mixed Poisson process (e.g. Grandell (1997)) is often assumed to study loss models incorporating time dependence such as IBNR and inflation models in the literature (e.g. Willmot (1989), Guo et al. (2013)), since this more general process can accommodate various realistic phenomena such as incurred claim seasonality, business growth, and heterogeneity of risk levels in the portfolio (e.g. see Willmot (1990) for further discussion). Also, Landriault et al. (2014) recently considered a non-homogeneous birth process for the claim counting process to study time-dependent aggregate claims incorporating inflation and payment delays into the model.

In this paper, our method involves not only multivariate distributions but also stochastic approach to incorporate the time-dependent claim sizes which are determined by the occurrence time of the event causing claims (losses). We then derive an expression for the joint moment of discounted compound renewal sum with time-dependent multivariate claims where there are possibly delays in reporting or payment. Concerning the claim incurral process, we assume that the number of events causing claims is a renewal process (i.e. arbitrary inter-occurrence time distribution) which contains the Poisson process as a special case. In this process, several works related to the discounted aggregate claims were done in the one dimensional case. For example, the expectation and higher moments of the discounted aggregate claims were studied by Léveillé and Garrido (2001a,b) and Léveillé et al. (2010). Furthermore, Woo and Cheung (2013) assumed certain dependent structure between the interclaim time and its resulting claim severity in this renewal process. Also, the joint moment of the discounted aggregate claim process with stochastic interest rate was obtained by Léveillé and Adékambi (2011). In light of particular emphasis on the payment delays with stochastic interest rate, the first two moments and the first joint moment of the aggregate discounted payments and expenses process for medical malpractice insurance were provided by Léveillé and Hamel (2013).

In the following section, the model assumptions and the expression for the joint moment of the $k$ types of discounted aggregate claims until time $t$ are provided. As a result, this proposed model can be applied to study some interesting insurance quantities involving the joint moments of the discounted aggregate claims, discounted reported/unreported aggregate claims as well as the number of IBNR claims under the effects of inflation in claim severities in Section 3. Consequently, for the Poisson and Erlang(2) claim incurral processes, covariance and correlation coefficient for two types of discounted aggregate claims, discounted aggregate reported/unreported claims and also total number of IBNR claims until various fixed times, are computed in Section 4. In particular, Izawa's bivariate gamma distribution (Izawa (1953)) for the joint distribution of two types of claim severities, is assumed. Finally, Section 5 presents some concluding remarks.

\section{The model}

The model assumptions and the main symbols used are as follows. To begin, the counting process of events causing losses/claims, denoted by $\left\{N_{t}\right\}_{t \geq 0}$, is a renewal process corresponding to a sequence of positive continuous independent and identically distributed (iid) random variables (rvs) $\left\{T_{i}\right\}_{i=1}^{\infty}$. Here $T_{i}$ is the occurrence time of the $i$-th event (assuming $T_{0}=0$ ), and $\tau_{i}=T_{i}-T_{i-1}$ is the interarrival time with common probability density function (pdf) $k(t)$, cumulative distribution function (cdf) $K(t)=1-\bar{K}(t)$ and Laplace transform $(\mathrm{LT}) \widetilde{k}(s)=\int_{0}^{\infty} e^{-s t} k(t) d t$. It is assumed that the $i$-th event which causes loss occurs at $T_{i}$ and contains $k$ types of claims (e.g. flood can concurrently damage several properties such as house and car). The $j$-type claim severity caused by the $i$-th event is denoted as $X_{i, j}$ where $\left\{X_{i, j}\right\}_{i=1}^{\infty}$ is a sequence of iid rvs with common cdf $P_{j}(y)$. A generic random vector of $k$ types of claims is denoted by $\mathbf{X}=\left(X_{1}, X_{2}, \ldots, X_{k}\right)$, and it has joint cdf $P_{\mathbf{X}}\left(x_{1}, x_{2}, \ldots, x_{k}\right)=\operatorname{Pr}\left\{X_{1} \leq\right.$ 
$\left.x_{1}, \ldots, X_{k} \leq x_{k}\right\}$ and joint moment generating function (mgf)

$$
M_{\mathbf{X}}(\mathbf{s})=E\left[e^{\mathbf{s X}^{\top}}\right]=E\left[e^{\sum_{j=1}^{k} s_{j} X_{j}}\right],
$$

where $\mathbf{s}=\left(s_{1}, s_{2}, \ldots, s_{k}\right)$ and $\mathbf{X}^{\top}$ is the transpose of $\mathbf{X}$. In addition, for each type of claim caused by the $i$-th event, there is delay in reporting or payment to be settled. This time lag for the $j$-type claim from $i$-th event is denoted by $L_{i, j}$, where $\left\{L_{i, j}\right\}_{i=1}^{\infty}$ is a sequence of iid rvs with common cdf $W_{j}(x)=1-\bar{W}_{j}(x)$, LT $\widetilde{w}_{j}(s)=\int_{0}^{\infty} e^{-s x} d W_{j}(x)$, and $\left\{L_{i, j}\right\}_{j=1}^{k}$ for fixed $i$ are independent. In other words, the time lag distribution is supposed to be different based on the type of claim. As $\mathbf{X}_{i}=\left(X_{i, 1}, X_{i, 2}, \ldots, X_{i, k}\right)$ represents the (baseline) claim sizes for $k$ types of claims from the $i$-th event, their costs are determined by some function $f_{t, T_{i}, L_{i, j}}(\cdot)$ implying possible dependence on the end of observation interval $t$, the occurrence time $T_{i}$, and the time lag $L_{i, j}$. Here we assume that $\left\{\mathbf{X}_{i}\right\}_{i=1}^{\infty},\left\{L_{i, j}\right\}_{i=1}^{\infty}$ and $\left\{T_{i}\right\}_{i=1}^{\infty}$ are mutually independent iid sequences. The force of interest at time $s$ is denoted as $\delta_{s}$, and the time-0 present value of $\$ 1$ payable at time $t$ is denoted as $D(t)=e^{-\int_{0}^{t} \delta_{s} d s}$. For the sake of simplicity we assume a constant force of interest $\delta$, i.e. $D(t)=e^{-\delta t}$ for $t \geq 0$ for the rest of paper.

For the $j$-type claim, let us now define the discounted total claim costs until time $t$ with reporting or payment delays as

$$
Z_{j}(t)=\sum_{i=1}^{N_{t}} D\left(T_{i}+L_{i, j}\right) f_{t, T_{i}, L_{i, j}}\left(X_{i, j}\right), \quad j=1,2, \ldots, k .
$$

Then the multivariate discounted total claim costs until time $t$ is

$$
\mathbf{Z}(t)=\left(Z_{1}(t), Z_{2}(t), \ldots, Z_{k}(t)\right), \quad t \geq 0,
$$

and its mgf is defined as

$$
M_{\mathbf{Z}(t)}(\mathbf{s})=E\left[e^{\mathbf{s Z}^{\top}(t)}\right], \quad t \geq 0,
$$

where $\mathbf{Z}^{\top}(t)$ is the transpose of $\mathbf{Z}(t)$. Then the mixed $\mathbf{n}=\left(n_{1}, n_{2}, \ldots, n_{k}\right)$-th moment about zero (or called $\mathbf{n}$-th joint moment) for $\left(Z_{1}(t), Z_{2}(t), \ldots, Z_{k}(t)\right)$ is

$$
E\left[\prod_{j=1}^{k} Z_{j}^{n_{j}}(t)\right]
$$

We note that its special case when $k=1$ is widely studied in the literature. As $n_{j}$ 's for $j=1,2, \ldots, k$, are possibly different, it is flexible to calculate the joint moment of different types of claims in any order. Also, it is possible to define joint cumulants where its generating function is defined by $\log M_{\mathbf{Z}(t)}(\mathbf{s})$. We can obtain (1) from the $\mathbf{n}$-th derivative of $M_{\mathbf{Z}(t)}(\mathbf{s})$ at $\mathbf{s}=\mathbf{0}$, i.e.

$$
M_{\mathbf{Z}(t)}^{(\mathbf{n})}(\mathbf{0})=\left.\left(\prod_{i=1}^{k} \frac{\partial^{n_{i}}}{\partial s_{i}^{n_{i}}}\right) M_{\mathbf{Z}(t)}(\mathbf{s})\right|_{\mathbf{s}=\mathbf{0}}, \quad n_{i}=1,2, \ldots,
$$

where $\mathbf{0}=(0,0, \ldots, 0)$ is a $1 \times k$ row vector. Note that from $\mathbf{Z}(t)$, several interesting quantities can be recovered with appropriate choices of $f(\cdot)$ (and $D(\cdot)$ ). Here are some examples as follows.

(i) If $f_{t, T_{i}, L_{i, j}}\left(X_{i, j}\right)=X_{i, j}$, then $\mathbf{Z}(t)$ is $k$ types of discounted aggregate claims process where there is delay in payment or settlement of the claims. See Léveillé and Hamel (2013) for $k=1$.

Further, assume $L_{i, j}=0$ (i.e. $D\left(T_{i}+L_{i, j}\right)=D\left(T_{i}\right)$ ), then $\mathbf{Z}(t)$ is $k$ types of discounted aggregate claim amounts until time $t$. See Section 3.1. In particular, numerous studies of this quantity for $k=1$ have been done under different claim counting processes. 
(ii) If $f_{t, T_{i}, L_{i, j}}\left(X_{i, j}\right)=X_{i, j} I\left(L_{i, j} \leq t-T_{i}\right)$, then $\mathbf{Z}(t)$ is $k$ types of discounted total reported claims until time $t$. See Section 3.2.

(iii) If $f_{t, T_{i}, L_{i, j}}\left(X_{i, j}\right)=X_{i, j} I\left(L_{i, j}>t-T_{i}\right)$, then $\mathbf{Z}(t)$ is $k$ types of discounted total IBNR (Incurred But Not Reported) claims until time $t$. See Section 3.3.

If we further assume $\delta_{s} \equiv 0$ and $X_{i, j} \equiv 1$, so that $f_{t, T_{i}, L_{i, j}}\left(X_{i, j}\right)=I\left(L_{i, j}>t-T_{i}\right)$ then $\mathbf{Z}(t)$ is $k$ types of total number of IBNR claims until time $t$. See Section 3.3.

For later use, let $M_{f_{t, T, L}(\mathbf{X})}(\mathbf{s})$ be the joint mgf of $f_{t, T, L}(\mathbf{X})$ defined by

$$
M_{f_{t, T, L}(\mathbf{X})}(\mathbf{s})=E\left[e^{\sum_{j=1}^{k} s_{j} f_{t, T, L_{j}}\left(X_{j}\right)}\right] .
$$

Here the convolution operator "*" is defined as $G * H(t)=\int_{0}^{t} G(t-y) d H(y)$ for functions $G$ and $H$. Then $G^{* i}$ denotes the $i$-th convolution of $G$ with itself such that $G^{* 0}(x)=I(x \geq 0), G^{* 1}(x) \equiv G(x)$, and $G^{* i}(x)=G^{*(i-1)} * G(x)$ for $i=2,3, \ldots$ In addition, we introduce the renewal function $m(t)=E\left[N_{t}\right]$ satisfying (e.g. Theorem 8.1.1 of Tijms (2003))

$$
m(t)=K(t)+\int_{0}^{t} K(t-y) d m(y)=\sum_{i=1}^{\infty} K^{* i}(t), \quad t \geq 0
$$

which plays an important role in the evaluation of (1). Moreover, we adopt the useful results from Lemma 4.1 and Lemma 4.2 of Léveillé and Garrido (2001a) as follows. Let us introduce a (possibly) defective distribution $H_{\delta}(t)=\int_{0}^{t} e^{-\delta y} d K(y)$ and $H_{\delta}^{* i}(t)=\int_{0}^{t} e^{-\delta y} d K^{* i}(y)$. Then from (2) we have $\sum_{i=1}^{\infty} H_{\delta}^{* i}(t)=\int_{0}^{t} e^{-\delta y} d m(y)$. As $t \rightarrow \infty$, it follows

$$
\sum_{i=1}^{\infty} H_{\delta}^{* i}(\infty)=\int_{0}^{\infty} e^{-\delta y} d m(y)=\frac{\widetilde{k}(\delta)}{1-\widetilde{k}(\delta)}
$$

Next, we shall derive the joint moment of $\mathbf{Z}(t)$ using its mgf. To begin, let $\mathcal{F}_{t}=\sigma\left\{N_{s} ; 0 \leq s \leq t\right\}$ be the smallest sigma field generated by $N_{s}$ for $0 \leq s \leq t$. Then by the double expectation formula, one finds

$$
M_{\mathbf{Z}(t)}(\mathbf{s})=E\left[E\left[e^{\mathbf{s} \mathbf{Z}^{\top}(t)} \mid \mathcal{F}_{t}\right]\right], \quad t \geq 0
$$

and the inner conditional expectation can be written as

$$
E\left[e^{\mathbf{s Z}^{\top}(t)} \mid \mathcal{F}_{t}\right]=\prod_{i=1}^{N_{t}} E\left[e^{\sum_{j=1}^{k} s_{j} e^{-\delta\left(T_{i}+L_{i, j}\right)} f_{t, T_{i}, L_{i, j}}\left(X_{i, j}\right)} \mid T_{i}\right] .
$$

\section{Applications}

In this section, with various choices of the forms of $f_{t, T_{i}, L_{i, j}}\left(X_{i, j}\right)$ and $L_{i, j}$ we derive the $\mathbf{n}$-th joint moments of $\mathbf{Z}(t)$ by using the preceding model.

\section{$3.1 \quad k$ types of discounted aggregate claims}

Setting $L_{i, j}=0$ and $f_{t, T_{i}, L_{i, j}}\left(X_{i, j}\right)=X_{i, j}$, one finds (5) as

$$
E\left[e^{\mathbf{s Z}^{\top}(t)} \mid \mathcal{F}_{t}\right]=\prod_{i=1}^{N_{t}} E\left[e^{\sum_{j=1}^{k} s_{j} e^{-\delta T_{i}} X_{i, j}} \mid T_{i}\right]
$$


and thus, from (4)

$$
M_{\mathbf{Z}(t)}(\mathbf{s})=E\left[\prod_{i=1}^{N_{t}} M_{\mathbf{X}}\left(e^{-\delta T_{i}} \mathbf{s}\right)\right]
$$

Now conditioning on the occurrence time of the first claim $\tau_{1}$, for $\tau_{1}=y \leq t$ implying $N_{t} \geq 1$, it follows that

$$
\begin{gathered}
E\left[\prod_{i=1}^{N_{t}} M_{\mathbf{X}}\left(e^{-\delta T_{i}} \mathbf{s}\right) \mid \tau_{1}=y\right]=M_{\mathbf{X}}\left(e^{-\delta y} \mathbf{s}\right) E\left[\prod_{i=2}^{N_{t}} M_{\mathbf{X}}\left(e^{-\delta y} e^{-\delta\left(\tau_{2}+\cdots+\tau_{i}\right)} \mathbf{s}\right) \mid \tau_{1}=y\right] \\
=M_{\mathbf{X}}\left(e^{-\delta y} \mathbf{s}\right) E\left[\prod_{i=1}^{N_{t-y}} M_{\mathbf{X}}\left(e^{-\delta y} e^{-\delta T_{i}} \mathbf{s}\right)\right]=M_{\mathbf{X}}\left(e^{-\delta y} \mathbf{s}\right) M_{\mathbf{Z}(t-y)}\left(e^{-\delta y} \mathbf{s}\right) .
\end{gathered}
$$

Thus, combining with the case of $\tau_{1}>t$ (i.e. $N_{t}=0$ ) results in

$$
M_{\mathbf{Z}(t)}(\mathbf{s})=\int_{t}^{\infty} d K(y)+\int_{0}^{t} M_{\mathbf{X}}\left(e^{-\delta y} \mathbf{s}\right) M_{\mathbf{Z}(t-y)}\left(e^{-\delta y} \mathbf{s}\right) d K(y) .
$$

Differentiating both sides $\mathbf{n}=\left(n_{1}, n_{2}, \ldots, n_{k}\right)$ times with respect to $\mathbf{s}=\left(s_{1}, s_{2}, \ldots, s_{k}\right)$ followed by inserting $\mathbf{s}=\mathbf{0}$ yields the $\mathbf{n}$-th joint moment of $\mathbf{Z}(t)$. Let us begin with the case of $\mathbf{n}=\mathbf{1}=(1,1, \ldots, 1)$ (i.e. $n_{i}=1$ for all $\left.i=1,2, \ldots, k\right)$. Define $\Omega=\{1,2, \ldots, k\}$ and $A$ is the set of all subsets of $\Omega$. Using the notation e.g $\partial^{3} f / \prod_{i \in\{1,2,3\}} \partial s_{i}$ for $\partial^{3} f / \partial s_{1} \partial s_{2} \partial s_{3}$, the first derivative of the product of $M_{\mathbf{X}}$ and $M_{\mathbf{Z}(t)}$ is

$$
\frac{\partial^{k} M_{\mathbf{X}}(\mathbf{s}) M_{\mathbf{Z}(t)}(\mathbf{s})}{\partial s_{1} \partial s_{2} \cdots \partial s_{k}}=\sum_{B \in A} \frac{\partial^{k-|B|} M_{\mathbf{X}}(\mathbf{s})}{\prod_{i \in B^{\complement}} \partial s_{i}} \cdot \frac{\partial^{|B|} M_{\mathbf{Z}(t)}(\mathbf{s})}{\prod_{i \in B} \partial s_{i}}
$$

where $B^{\complement}$ is the complement of set $B$ and $|B|$ denotes the number of elements in set $B$ (e.g. Proposition 5 of Hardy (2006)), and thus one finds

$$
\left.\frac{\partial^{k} M_{\mathbf{X}}\left(e^{-\delta y} \mathbf{s}\right) M_{\mathbf{Z}(t)}\left(e^{-\delta y} \mathbf{s}\right)}{\partial s_{1} \partial s_{2} \cdots \partial s_{k}}\right|_{\mathbf{s}=\mathbf{0}}=\left.e^{-k \delta y} \sum_{B \in A} \mu_{B^{\complement}} \frac{\partial^{|B|} M_{\mathbf{Z}(t)}(\mathbf{s})}{\prod_{i \in B} \partial s_{i}}\right|_{\mathbf{s}=\mathbf{0}},
$$

where for set $\Upsilon$,

$$
\mu_{\Upsilon}=E\left[\prod_{i \in \Upsilon} X_{i}\right]
$$

(e.g. $\mu_{\{1,3\}}=E\left[X_{1} X_{3}\right]$ ). By separating the term when $B=\Omega$ is the universal set, the expression on the right-hand side of $(7)$ can be rewritten as

$$
e^{-k \delta y} M_{\mathbf{Z}(t)}^{(\mathbf{1})}(\mathbf{0})+\left.e^{-k \delta y} \sum_{B \in A \backslash \Omega} \mu_{B^{\complement}} \frac{\partial^{|B|} M_{\mathbf{Z}(t)}(\mathbf{s})}{\prod_{i \in B} \partial s_{i}}\right|_{\mathbf{s}=\mathbf{0}} .
$$

Then the first derivative of (6) evaluated at $\mathbf{0}$ with the help of (9) is a renewal equation given by

$$
M_{\mathbf{Z}(t)}^{(\mathbf{1})}(\mathbf{0})=a_{\mathbf{1}}(t)+M_{\mathbf{Z}(\cdot)}^{(\mathbf{1})}(\mathbf{0}) * H_{k \delta}(t), \quad t \geq 0,
$$

with $H_{k \delta}(t)=\int_{0}^{t} e^{-k \delta y} d K(y)$ and non-homogeneous term

$$
a_{\mathbf{1}}(t)=\left.\sum_{B \in A \backslash \Omega} \mu_{B^{\complement}} \int_{0}^{t} e^{-k \delta y} \frac{\partial^{|B|} M_{\mathbf{Z}(t-y)}(\mathbf{s})}{\prod_{i \in B} \partial s_{i}}\right|_{\mathbf{s}=\mathbf{0}} d K(y)=\left.\sum_{B \in A \backslash \Omega} \mu_{B^{\complement}} \frac{\partial^{|B|} M_{\mathbf{Z}(\cdot)}(\mathbf{s})}{\prod_{i \in B} \partial s_{i}}\right|_{\mathbf{s}=\mathbf{0}} * H_{k \delta}(t) .
$$


It is known that the solution of (10) is given by

$$
M_{\mathbf{Z}(t)}^{(\mathbf{1})}(\mathbf{0})=a_{\mathbf{1}} * \sum_{i=0}^{\infty} H_{k \delta}^{* i}(t), \quad t \geq 0,
$$

where $H_{k \delta}^{* i}(t)=\int_{0}^{t} e^{-k \delta y} d K^{* i}(y)$ (see Lemma 4.1 of Léveillé and Garrido (2001a) when $k=1$ ). Applying the result given by Léveillé and Garrido (2001a) with the use of the renewal function $m(t)=E\left[N_{t}\right]=$ $\sum_{i=1}^{\infty} K^{* i}(t),(12)$ can be expressed as

$$
E\left[\prod_{j=1}^{k} Z_{j}(t)\right]=M_{\mathbf{Z}(t)}^{(\mathbf{1})}(\mathbf{0})=a_{\mathbf{1}}(t)+\int_{0}^{t} e^{-k \delta y} a_{\mathbf{1}}(t-y) d m(y), \quad t \geq 0
$$

where $a_{\mathbf{1}}(t)$ is given in (11). Equivalently,

$$
E\left[\prod_{j=1}^{k} Z_{j}(t)\right]=\sum_{B \in A \backslash \Omega} \mu_{B^{\complement}} \int_{0}^{t} e^{-k \delta y} E\left[\prod_{j \in B} Z_{j}(t-y)\right] d m(y) .
$$

In particular, for univariate case (i.e. $k=1$ ), $a_{\mathbf{1}}(t)$ in (11) simply becomes $\mu_{1} H_{\delta}(t)$. Consequently, from (12) or (13) we have

$$
E\left[Z_{1}(t)\right]=E\left[X_{1}\right] \sum_{i=1}^{\infty} H_{\delta}^{* i}(t)=E\left[X_{1}\right] \int_{0}^{t} e^{-\delta y} d m(y), \quad t \geq 0,
$$

which is consistent with Theorem 4.1 of Léveillé and Garrido (2001a).

Similarly, for a general $n_{i} \geq 0$, using the result in Proposition 6 of Hardy (2006), the $\mathbf{n}$-th derivative of the product of $M_{\mathbf{X}}$ and $M_{\mathbf{Z}(t)}$ is given by

$$
\frac{\partial^{\sum_{i=1}^{k} n_{i}} M_{\mathbf{X}}(\mathbf{s}) M_{\mathbf{Z}(t)}(\mathbf{s})}{\partial s_{1}^{n_{1}} \partial s_{2}^{n_{2}} \cdots \partial s_{k}^{n_{k}}}=\sum_{l_{1}=0}^{n_{1}} \sum_{l_{2}=0}^{n_{2}} \cdots \sum_{l_{k}=0}^{n_{k}}\left(\begin{array}{c}
n_{1} \\
l_{1}
\end{array}\right)\left(\begin{array}{c}
n_{2} \\
l_{2}
\end{array}\right) \cdots\left(\begin{array}{c}
n_{k} \\
l_{k}
\end{array}\right) \frac{\partial^{\sum_{j=1}^{k}\left(n_{j}-l_{j}\right)} M_{\mathbf{X}}(\mathbf{s})}{\prod_{j=1}^{k} \partial s_{j}^{n_{j}-l_{j}}} \cdot \frac{\partial^{\sum_{j=1}^{k} l_{j}} M_{\mathbf{Z}(t)}(\mathbf{s})}{\prod_{j=1}^{k} \partial s_{j}^{l_{j}}} .
$$

Hence the $\mathbf{n}$-th partial derivative of $M_{\mathbf{X}}\left(e^{-\delta y} \mathbf{s}\right) M_{\mathbf{Z}(t)}\left(e^{-\delta y} \mathbf{s}\right)$ with substitution of $\mathbf{s}=\mathbf{0}$ followed by isolating the final term in $k$ summations (i.e. when $l_{j}=n_{j}$ for all $j=1,2, \ldots, k$ ) can be found as

$$
e^{-\delta y \sum_{i=1}^{k} n_{i}} M_{\mathbf{Z}(t)}^{(\mathbf{n})}(\mathbf{0})+\left.e^{-\delta y \sum_{i=1}^{k} n_{i}} \sum_{G}\left(\begin{array}{c}
n_{1} \\
l_{1}
\end{array}\right) \cdots\left(\begin{array}{c}
n_{k} \\
l_{k}
\end{array}\right) \frac{\partial^{\sum_{j=1}^{k}\left(n_{j}-l_{j}\right)} M_{\mathbf{X}}(\mathbf{s})}{\prod_{j=1}^{k} \partial s_{j}^{n_{j}-l_{j}}} \cdot \frac{\partial^{\sum_{j=1}^{k} l_{j}} M_{\mathbf{Z}(t)}(\mathbf{s})}{\prod_{j=1}^{k} \partial s_{j}^{l_{j}}}\right|_{\mathbf{s}=\mathbf{0}},
$$

where

$$
G=\left\{\left(l_{1}, l_{2}, \ldots, l_{k}\right) \mid 0 \leq l_{i} \leq n_{i}, \text { for } i=1,2, \ldots, k\right\} \backslash\left(n_{1}, n_{2}, \ldots, n_{k}\right) .
$$

This leads to the following theorem.

Theorem 1 For $n_{i}=0,1,2, \ldots$, and $\sum_{i=1}^{k} n_{i} \neq 0$, the $\mathbf{n}$-th joint moment of $k$ types of discounted aggregate claims until $t$ satisfies the renewal equation as

$$
E\left[\prod_{j=1}^{k} Z_{j}^{n_{j}}(t)\right]=M_{\mathbf{Z}(t)}^{(\mathbf{n})}(\mathbf{0})=a_{\mathbf{n}}(t)+M_{\mathbf{Z}(\cdot)}^{(\mathbf{n})}(\mathbf{0}) * H_{\eta \delta}(t), \quad t \geq 0
$$


where $\sum_{i=1}^{k} n_{i}=\eta$. Equivalently, one has

$$
E\left[\prod_{j=1}^{k} Z_{j}^{n_{j}}(t)\right]=a_{\mathbf{n}}(t)+\int_{0}^{t} e^{-\eta \delta y} a_{\mathbf{n}}(t-y) d m(y), \quad t \geq 0
$$

where

$$
a_{\mathbf{n}}(t)=\sum_{G}\left(\begin{array}{c}
n_{1} \\
l_{1}
\end{array}\right) \cdots\left(\begin{array}{c}
n_{k} \\
l_{k}
\end{array}\right) E\left[\prod_{j=1}^{k} X_{j}^{n_{j}-l_{j}}\right] \int_{0}^{t} e^{-\eta \delta y} E\left[\prod_{j=1}^{k} Z_{j}^{l_{j}}(t-y)\right] d K(y),
$$

with $G$ given in (15). Also, with (18), (17) can be expressed as

$$
E\left[\prod_{j=1}^{k} Z_{j}^{n_{j}}(t)\right]=\sum_{G}\left(\begin{array}{c}
n_{1} \\
l_{1}
\end{array}\right) \cdots\left(\begin{array}{c}
n_{k} \\
l_{k}
\end{array}\right) E\left[\prod_{j=1}^{k} X_{j}^{n_{j}-l_{j}}\right] \int_{0}^{t} e^{-\eta \delta y} E\left[\prod_{j=1}^{k} Z_{j}^{l_{j}}(t-y)\right] d m(y) .
$$

Proof: The proof is similar to the case of $n_{i}=1$ for all $i=1,2, \ldots, k$ derived previously. From (6), differentiating both sides $\mathbf{n}=\left(n_{1}, n_{2}, \ldots, n_{k}\right)$ times with respect to $\mathbf{s}=\left(s_{1}, s_{2}, \ldots, s_{k}\right)$ results in

$$
\left(\prod_{i=1}^{k} \frac{\partial^{n_{i}}}{\partial s_{i}^{n_{i}}}\right) M_{\mathbf{Z}(t)}(\mathbf{s})=\int_{0}^{t} \frac{\partial^{\sum_{i=1}^{k} n_{i}} M_{\mathbf{X}}\left(e^{-\delta y} \mathbf{s}\right) M_{\mathbf{Z}(t-y)}\left(e^{-\delta y} \mathbf{s}\right)}{\prod_{i=1}^{k} \partial s_{i}^{n_{i}}} d K(y),
$$

and setting $\mathbf{s}=\mathbf{0}$ with the help of (14) yields

$$
\begin{aligned}
M_{\mathbf{Z}(t)}^{(\mathbf{n})}(\mathbf{0})= & \int_{0}^{t} e^{-\eta \delta y} M_{\mathbf{Z}(t-y)}^{(\mathbf{n})}(\mathbf{0}) d K(y)+\sum_{G}\left(\begin{array}{c}
n_{1} \\
l_{1}
\end{array}\right) \cdots\left(\begin{array}{c}
n_{k} \\
l_{k}
\end{array}\right) \\
& \times\left.\int_{0}^{t} e^{-\eta \delta y} \frac{\partial^{\sum_{j=1}^{k}\left(n_{j}-l_{j}\right)} M_{\mathbf{X}}(\mathbf{s})}{\prod_{j=1}^{k} \partial s_{j}^{n_{j}-l_{j}}} \cdot \frac{\partial^{\sum_{j=1}^{k} l_{j}} M_{\mathbf{Z}(t-y)}(\mathbf{s})}{\prod_{j=1}^{k} \partial s_{j}^{l_{j}}}\right|_{\mathbf{s}=\mathbf{0}} d K(y) .
\end{aligned}
$$

But $M_{\mathbf{Z}(t)}^{(\mathbf{n})}(\mathbf{0})=E\left[\prod_{j=1}^{k} Z_{j}^{n_{j}}(t)\right]$ and $M_{\mathbf{X}(t)}^{(\mathbf{n})}(\mathbf{0})=E\left[\prod_{j=1}^{k} X_{j}^{n_{j}}(t)\right]$, so the above equation becomes

$$
\begin{aligned}
E\left[\prod_{j=1}^{k} Z_{j}^{n_{j}}(t)\right]= & \int_{0}^{t} E\left[\prod_{j=1}^{k} Z_{j}^{n_{j}}(t-y)\right] d H_{\eta \delta}(y)+\sum_{G}\left(\begin{array}{c}
n_{1} \\
l_{1}
\end{array}\right) \cdots\left(\begin{array}{c}
n_{k} \\
l_{k}
\end{array}\right) E\left[\prod_{j=1}^{k} X_{j}^{n_{j}-l_{j}}\right] \\
& \times \int_{0}^{t} E\left[\prod_{j=1}^{k} Z_{j}^{l_{j}}(t-y)\right] d H_{\eta \delta}(y),
\end{aligned}
$$

where $\sum_{i=1}^{k} n_{i}=\eta$ and $H_{\delta}(t)=\int_{0}^{t} e^{-\delta y} d K(y)$. Hence, the result in (16) with (18) follows.

Alternatively, similar to (12), using the solution of the renewal equation (16), it can be expressed as

$$
E\left[\prod_{j=1}^{k} Z_{j}^{n_{j}}(t)\right]=a_{\mathbf{n}} * \sum_{i=0}^{\infty} H_{\eta \delta}^{* i}(t), \quad t \geq 0,
$$

where $H_{k \delta}^{* i}(t)=\int_{0}^{t} e^{-k \delta y} d K^{* i}(y)$. Therefore, using the solution of renewal function $m(t)=\sum_{i=1}^{\infty} K^{* i}(t)$ leads to (17).

Note that when $k=1$, Theorem 1 reduces to the result in Theorem 2.1 of Léveillé and Garrido (2001b) or Proposition 2.1 of Woo and Cheung (2013) for the time-independent claim case. 
Example $1\left(k=2\right.$ and $\left.n_{1}=n_{2}=2\right)$ The joint second moment of two types of discounted aggregate claims until time $t$ is given as follows. In this case, (15) is $G=\left\{\left(l_{1}, l_{2}\right) \mid l_{i}=0,1,2\right.$, for $\left.i=1,2\right\} \backslash(2,2)$. Then from (19), one finds

$$
\begin{aligned}
E & {\left[Z_{1}^{2}(t) Z_{2}^{2}(t)\right]=E\left[X_{1}^{2} X_{2}^{2}\right] \int_{0}^{t} e^{-4 \delta y} d m(y)+2 E\left[X_{1}^{2} X_{2}\right] \int_{0}^{t} e^{-4 \delta y} E\left[Z_{2}(t-y)\right] d m(y) } \\
& +E\left[X_{1}^{2}\right] \int_{0}^{t} e^{-4 \delta y} E\left[Z_{2}^{2}(t-y)\right] d m(y)+2 E\left[X_{1} X_{2}^{2}\right] \int_{0}^{t} e^{-4 \delta y} E\left[Z_{1}(t-y)\right] d m(y) \\
& +4 E\left[X_{1} X_{2}\right] \int_{0}^{t} e^{-4 \delta y} E\left[Z_{1}(t-y) Z_{2}(t-y)\right] d m(y)+2 E\left[X_{1}\right] \int_{0}^{t} e^{-4 \delta y} E\left[Z_{1}(t-y) Z_{2}^{2}(t-y)\right] d m(y) \\
& +E\left[X_{2}^{2}\right] \int_{0}^{t} e^{-4 \delta y} E\left[Z_{1}^{2}(t-y)\right] d m(y)+2 E\left[X_{2}\right] \int_{0}^{t} e^{-4 \delta y} E\left[Z_{1}^{2}(t-y) Z_{2}(t-y)\right] d m(y),
\end{aligned}
$$

and $E\left[Z_{1}(t) Z_{2}(t)\right]$ can be evaluated from (13) when $k=2$ as

$$
E\left[Z_{1}(t) Z_{2}(t)\right]=\int_{0}^{t} e^{-2 \delta y}\left\{E\left[X_{1} X_{2}\right]+E\left[X_{1}\right] E\left[Z_{2}(t-y)\right]+E\left[X_{2}\right] E\left[Z_{1}(t-y)\right]\right\} d m(y) .
$$

Also, $E\left[Z_{1}^{2}(t) Z_{2}(t)\right]$ can be obtained by setting $n_{1}=2$ and $n_{2}=1$ in (19). That is,

$$
\begin{aligned}
E\left[Z_{1}^{2}(t) Z_{2}(t)\right]= & \int_{0}^{t} e^{-3 \delta y}\left\{E\left[X_{1}^{2} X_{2}\right]+E\left[X_{1}^{2}\right] E\left[Z_{2}(t-y)\right]+2 E\left[X_{1} X_{2}\right] E\left[Z_{1}(t-y)\right]\right. \\
& \left.+2 E\left[X_{1}\right] E\left[Z_{1}(t-y) Z_{2}(t-y)\right]+E\left[X_{2}\right] E\left[Z_{1}^{2}(t-y)\right]\right\} d m(y) .
\end{aligned}
$$

Similarly, $E\left[Z_{1}(t) Z_{2}^{2}(t)\right]$ is evaluated with $n_{1}=1$ and $n_{2}=2$ in (19). We remark that all integrals involved in the evaluation of $E\left[Z_{1}^{2}(t) Z_{2}^{2}(t)\right]$ as given above are in terms of $E\left[Z_{i}(t)\right]$ and $E\left[Z_{i}^{2}(t)\right]$ which are well-studied in the literature (e.g. Léveillé and Garrido (2001a,b)). Especially, if $N_{t}$ is assumed to be the (mixed) Poisson or Erlang(2) renewal process, it can be calculated via straightforward (but tedious) integration.

When $t \rightarrow \infty$ and $\delta>0$, from (19) with the help of (3), the asymptotic joint moment of $k$ types of discounted aggregate claims is recursively obtained as

$$
E\left[\prod_{j=1}^{k} Z_{j}^{n_{j}}(\infty)\right]=\sum_{G}\left(\begin{array}{c}
n_{1} \\
l_{1}
\end{array}\right) \cdots\left(\begin{array}{c}
n_{k} \\
l_{k}
\end{array}\right) E\left[\prod_{j=1}^{k} X_{j}^{n_{j}-l_{j}}\right] E\left[\prod_{j=1}^{k} Z_{j}^{l_{j}}(\infty)\right] \frac{\widetilde{k}(\eta \delta)}{1-\widetilde{k}(\eta \delta)} .
$$

Clearly, when $k=1,(20)$ agrees with the one obtained by Léveillé and Garrido (2001b, Corollary 2.1).

\section{$3.2 k$ types of discounted aggregate reported claims}

Let us consider that there is a reporting delay until the insurer observes claims (or it can be regarded as the case where there is a delay for claim to be settled). Thus, we assume $L_{i, j}>0$ and $f_{t, T_{i}, L_{i, j}}\left(X_{i, j}\right)=$ $X_{i, j} I\left(L_{i, j} \leq t-T_{i}\right)$ for $t \geq 0$. Conditioning on $L_{i, j}=v_{j}$ for $j=1,2, \ldots, k$ leads the inner expectation of $(5)$ as

$$
\begin{aligned}
& E\left[e^{\sum_{j=1}^{k} s_{j} e^{-\delta\left(T_{i}+L_{i, j}\right)} f_{t, T_{i}, L_{i, j}}\left(X_{i, j}\right)} \mid T_{i}\right] \\
& \quad=\int_{0}^{\infty} \cdots \int_{0}^{\infty} E\left[e^{\sum_{j=1}^{k} s_{j} e^{-\delta\left(T_{i}+L_{i, j}\right)} X_{i, j} I\left(L_{i, j} \leq t-T_{i}\right)} \mid L_{i, 1}=v_{1}, \ldots, L_{i, k}=v_{k}, T_{i}\right] d W_{1}\left(v_{1}\right) \cdots d W_{k}\left(v_{k}\right) .
\end{aligned}
$$


Let us define

$$
M_{t, \mathbf{X}}(\mathbf{s})=\int_{0}^{\infty} \cdots \int_{0}^{\infty} E\left[e^{\sum_{j=1}^{k} s_{j} e^{-\delta L_{i, j} X_{i, j} I\left(L_{i, j} \leq t\right)}} \mid L_{i, 1}=v_{1}, \ldots, L_{i, k}=v_{k}\right] d W_{1}\left(v_{1}\right) \cdots d W_{k}\left(v_{k}\right) .
$$

Then from (4) and (5), using (21) and (22) we obtain

$$
M_{\mathbf{Z}(t)}(\mathbf{s})=E\left[\prod_{i=1}^{N_{t}} M_{t-T_{i}, \mathbf{X}}\left(e^{-\delta T_{i}} \mathbf{s}\right)\right] .
$$

Again conditioning on $\tau_{1}=y<t$ yields

$$
\begin{aligned}
E & {\left[\prod_{i=1}^{N_{t}} M_{t-T_{i}, \mathbf{X}}\left(e^{-\delta T_{i}} \mathbf{s}\right) \mid \tau_{1}=y\right] } \\
& =M_{t-y, \mathbf{X}}\left(e^{-\delta y} \mathbf{s}\right) E\left[\prod_{i=2}^{N_{t}} M_{t-y-\left(\tau_{2}+\cdots+\tau_{i}\right), \mathbf{X}}\left(e^{-\delta y} e^{-\delta\left(\tau_{2}+\cdots+\tau_{i}\right)} \mathbf{s}\right) \mid \tau_{1}=y\right] \\
& =M_{t-y, \mathbf{X}}\left(e^{-\delta y} \mathbf{s}\right) E\left[\prod_{i=1}^{N_{t-y}} M_{t-y-T_{i}, \mathbf{X}}\left(e^{-\delta y} e^{-\delta T_{i}} \mathbf{s}\right)\right]=M_{t-y, \mathbf{X}}\left(e^{-\delta y} \mathbf{s}\right) M_{\mathbf{Z}(t-y)}\left(e^{-\delta y} \mathbf{s}\right),
\end{aligned}
$$

and hence putting together with the case of $\tau_{1}>t$ results in

$$
M_{\mathbf{Z}(t)}(\mathbf{s})=\int_{t}^{\infty} d K(y)+\int_{0}^{t} M_{t-y, \mathbf{X}}\left(e^{-\delta y} \mathbf{s}\right) M_{\mathbf{Z}(t-y)}\left(e^{-\delta y} \mathbf{s}\right) d K(y) .
$$

Then differentiating both sides of (23) $\mathbf{n}$ times with respect to $\mathbf{s}$ followed by putting $\mathbf{s}=\mathbf{0}$ leads us to the $\mathbf{n}$-th joint moment of two types of discounted aggregate reported claims until $t$. In what follows, it is convenient to introduce the function

$$
\omega_{\delta, j}(t)=\int_{0}^{t} e^{-\delta v} d W_{j}(v),
$$

which is a (possibly) defective distribution. When $\mathbf{n}=\mathbf{1}$ (i.e. $n_{i}=1$ for all $i=1,2, \ldots, k$ ), analogous to (9), the first derivative of the product of $M_{t, \mathbf{X}}$ and $M_{\mathbf{Z}(t)}$ at $\mathbf{s}=\mathbf{0}$ is obtained as follows. First, from (22), when $k=2$ one has

$$
\left.\sum_{B \in A \backslash\{1,2\}} \frac{\partial^{2-|B|} M_{t, \mathbf{X}}(\mathbf{s})}{\prod_{i \in B^{\complement}} \partial s_{i}}\right|_{\mathbf{s}=\mathbf{0}}=E\left[X_{1} X_{2}\right] \omega_{\delta, 1}(t) \omega_{\delta, 2}(t)+E\left[X_{2}\right] \omega_{\delta, 2}(t)+E\left[X_{1}\right] \omega_{\delta, 1}(t) .
$$

Then for the general case $k \geq 1$, it follows that

$$
\left.\sum_{B \in A \backslash \Omega} \frac{\partial^{k-|B|} M_{t, \mathbf{X}}(\mathbf{s})}{\prod_{i \in B^{\complement}} \partial s_{i}}\right|_{\mathbf{s}=\mathbf{0}}=\sum_{B \in A \backslash \Omega} \mu_{B^{\complement}}(t),
$$

where for set $\Upsilon$,

$$
\mu_{\Upsilon}(t)=\mu_{\Upsilon} \prod_{i \in \Upsilon} \omega_{\delta, i}(t),
$$

(e.g. $\left.\mu_{\{1,2\}}(t)=E\left[X_{1} X_{2}\right] \omega_{\delta, 1}(t) \omega_{\delta, 2}(t)\right)$ and $\mu_{\Upsilon}$ is given in (8). Therefore, we arrive at

$$
\left.\frac{\partial^{k} M_{t, \mathbf{X}}\left(e^{-\delta y} \mathbf{s}\right) M_{\mathbf{Z}(t)}\left(e^{-\delta y} \mathbf{s}\right)}{\partial s_{1} \partial s_{2} \cdots \partial s_{k}}\right|_{\mathbf{s}=\mathbf{0}}=e^{-k \delta y} M_{\mathbf{Z}(t)}^{(\mathbf{1})}(\mathbf{0})+\left.e^{-k \delta y} \sum_{B \in A \backslash \Omega} \mu_{B^{\complement}}(t) \frac{\partial^{|B|} M_{\mathbf{Z}(t)}(\mathbf{s})}{\prod_{i \in B} \partial s_{i}}\right|_{\mathbf{s}=\mathbf{0}},
$$


and in turn, from (23) the joint expectation of $k$ types of discounted aggregate reported claims satisfies the renewal equation

$$
M_{\mathbf{Z}(t)}^{(\mathbf{1})}(\mathbf{0})=r_{\mathbf{1}}(t)+M_{\mathbf{Z}(\cdot)}^{(\mathbf{1})}(\mathbf{0}) * H_{k \delta}(t)
$$

where

$$
r_{\mathbf{1}}(t)=\left.\sum_{B \in A \backslash \Omega} \int_{0}^{t} e^{-k \delta y} \mu_{B^{\mathrm{C}}}(t-y) \frac{\partial^{|B|} M_{\mathbf{Z}(t-y)}(\mathbf{s})}{\prod_{i \in B} \partial s_{i}}\right|_{\mathbf{s}=\mathbf{0}} d K(y) .
$$

Then (26) has the solution

$$
M_{\mathbf{Z}(t)}^{(\mathbf{1})}(\mathbf{0})=r_{\mathbf{1}} * \sum_{i=0}^{\infty} H_{k \delta}^{* i}(t), \quad t \geq 0
$$

or equivalently

$$
E\left[\prod_{j=1}^{k} Z_{j}(t)\right]=M_{\mathbf{Z}(t)}^{(\mathbf{1})}(\mathbf{0})=r_{\mathbf{1}}(t)+\int_{0}^{t} e^{-k \delta y} r_{\mathbf{1}}(t-y) d m(y)
$$

where $m(t)=E\left[N_{t}\right]$ is the renewal function. Similar to (13), with (27), it can be also written as

$$
E\left[\prod_{j=1}^{k} Z_{j}(t)\right]=\sum_{B \in A \backslash \Omega} \int_{0}^{t} e^{-k \delta y} \mu_{B^{\mathrm{c}}}(t-y) E\left[\prod_{j \in B} Z_{j}(t-y)\right] d m(y) .
$$

Example $2\left(k=1\right.$ and $\left.n_{1}=1\right)$ Using (25) with (24), (27) reduces to

$$
r_{1}(t)=E\left[X_{1}\right] \int_{0}^{t} e^{-\delta y} \omega_{\delta, 1}(t-y) d K(y)=E\left[X_{1}\right]\left[\omega_{\delta, 1} * H_{\delta}(t)\right],
$$

and then from (28), the mean of the discounted aggregate reported claim until $t$ is given by

$$
E\left[Z_{1}(t)\right]=E\left[X_{1}\right]\left[\omega_{\delta, 1} * H_{\delta} * \sum_{i=0}^{\infty} H_{\delta}^{* i}(t)\right]=E\left[X_{1}\right] \int_{0}^{t} e^{-\delta y} \omega_{\delta, 1}(t-y) d m(y), \quad t \geq 0 .
$$

In particular, if $\delta=0$ then from (24) and (29), $r_{1}(t)=\mu_{\{1\}}\left[W_{1} * K(t)\right]$ and thus

$$
E\left[Z_{1}(t)\right]=E\left[X_{1}\right] \int_{0}^{t} W_{1}(t-y) d m(y)=E\left[X_{1}\right] m_{d}(t),
$$

where $m_{d}(t)=\sum_{i=1}^{\infty} W_{1} * K^{* i}(t)$ is the renewal function for a delayed renewal process $\left\{N_{d, t}\right\}_{t \geq 0}$ corresponding to the ordinary renewal process $\left\{N_{t}\right\}_{t \geq 0}$ with the different interarrival distribution for the first event as $W_{1} * K(t)$ (e.g. Lemma 6.1 of Léveillé and Garrido (2001a)). We remark that the mean of the number of reported claims until $t$ appearing in (30) (i.e. excluding $\mu_{1}$ ) is also given by Karlsson (1974, p.384) as

$$
\sum_{i=0}^{\infty}\left(W * K_{0} * K^{* i}\right)(t),
$$

where $W$ is a lag cdf, $K_{0}$ is the interarrival cdf of the first claim starting from time 0 (in the present paper $K_{0}$ is assumed to be $K$ ).

For a general $n_{i} \geq 0$, the following result holds. 
Theorem 2 For $n_{i}=0,1,2, \ldots$, and $\sum_{i=1}^{k} n_{i} \neq 0$, the $\mathbf{n}$-th joint moment of $k$ types of discounted aggregate reported claims until $t$ satisfies the renewal equation

$$
E\left[\prod_{j=1}^{k} Z_{j}^{n_{j}}(t)\right]=M_{\mathbf{Z}(t)}^{(\mathbf{n})}(\mathbf{0})=r_{\mathbf{n}}(t)+M_{\mathbf{Z}(t)}^{(\mathbf{n})}(\mathbf{0}) * H_{\eta \delta}(t), \quad t \geq 0
$$

where $\eta=\sum_{i=1}^{k} n_{i}$, which can be evaluated recursively using the equation

$$
E\left[\prod_{j=1}^{k} Z_{j}^{n_{j}}(t)\right]=r_{\mathbf{n}}(t)+\int_{0}^{t} e^{-\eta \delta y} r_{\mathbf{n}}(t-y) d m(y), \quad t \geq 0
$$

where

$$
r_{\mathbf{n}}(t)=\sum_{G}\left(\begin{array}{l}
n_{1} \\
l_{1}
\end{array}\right) \cdots\left(\begin{array}{l}
n_{k} \\
l_{k}
\end{array}\right) E\left[\prod_{j \in C} X_{j}^{n_{j}-l_{j}}\right] \int_{0}^{t} e^{-\eta \delta y} E\left[\prod_{j=1}^{k} Z_{j}^{l_{j}}(t-y)\right]\left[\prod_{j \in C} \omega_{\left(n_{j}-l_{j}\right) \delta, j}(t-y)\right] d K(y),
$$

with $G$ given in (15), $C=C_{l_{1}, \ldots, l_{k}}$ being a subset of $\{1,2, \ldots, k\}$ containing the $i$ 's such that $l_{i} \neq n_{i}$, and $\omega_{\delta, j}(t)$ given in (24). Equivalently,

$E\left[\prod_{j=1}^{k} Z_{j}^{n_{j}}(t)\right]=\sum_{G}\left(\begin{array}{c}n_{1} \\ l_{1}\end{array}\right) \cdots\left(\begin{array}{c}n_{k} \\ l_{k}\end{array}\right) E\left[\prod_{j \in C} X_{j}^{n_{j}-l_{j}}\right] \int_{0}^{t} e^{-\eta \delta y} E\left[\prod_{j=1}^{k} Z_{j}^{l_{j}}(t-y)\right]\left[\prod_{j \in C} \omega_{\left(n_{j}-l_{j}\right) \delta, j}(t-y)\right] d m(y)$.

Proof: Using the result

$$
\left.\frac{\partial^{\sum_{j=1}^{k}\left(n_{j}-l_{j}\right)} M_{t, \mathbf{X}}(\mathbf{s})}{\prod_{j=1}^{k} \partial s_{j}^{n_{j}-l_{j}}}\right|_{\mathbf{s}=\mathbf{0}}=E\left[\prod_{i \in C} X_{i}^{n_{i}-l_{i}}\right] \prod_{i \in C} \omega_{\left(n_{i}-l_{i}\right) \delta, i}(t),
$$

the rest of proof is similar to the one for $\mathbf{n}=\mathbf{1}$ given previously.

Moreover, from (31) with (24) and (3) when $t \rightarrow \infty$, the asymptotic joint moment of $k$ types of discounted aggregate reported claims can be obtained in the following corollary.

Corollary 1 For $n_{i}=0,1,2, \ldots$, and $\sum_{i=1}^{k} n_{i} \neq 0$, the asymptotic $\mathbf{n}$-th joint moment of $k$ types of discounted aggregate reported claims is given by

$$
E\left[\prod_{j=1}^{k} Z_{j}^{n_{j}}(\infty)\right]=\sum_{G}\left(\begin{array}{c}
n_{1} \\
l_{1}
\end{array}\right) \cdots\left(\begin{array}{c}
n_{k} \\
l_{k}
\end{array}\right) E\left[\prod_{j \in C} X_{j}^{n_{j}-l_{j}}\right] E\left[\prod_{j=1}^{k} Z_{j}^{l_{j}}(\infty)\right]\left[\prod_{j \in C} \widetilde{w}_{j}\left\{\left(n_{j}-l_{j}\right) \delta\right\}\right] \frac{\widetilde{k}(\eta \delta)}{1-\widetilde{k}(\eta \delta)} .
$$

Remark 1 If there no reporting delay (i.e. for $x \geq 0$ and $j=1,2, \ldots, k, W_{j}(x)=\operatorname{Pr}\left(L_{i, j}=0\right)=1$ ), then we can retrieve results obtained in Section 3.1. 


\section{$3.3 \quad k$ types of discounted total IBNR claims}

Since each incurred claim $X_{i, j}$ is either a reported claim or an IBNR claim, the joint moment of $k$ types of discounted total IBNR claims can be derived in the same manner as for the model in Section 3.2. Specifically, we only need to impose a slightly different assumption on $f_{t, T_{i}, L_{i, j}}\left(X_{i, j}\right)$, namely a reverse inequality on the event in the indicator function $I\left(L_{i, j}>t-T_{i}\right)$ for $t \geq 0$. In this case, (22) is now updated as

$$
M_{t, \mathbf{X}}^{*}(\mathbf{s})=\int_{0}^{\infty} \cdots \int_{0}^{\infty} E\left[e^{\sum_{j=1}^{k} s_{j} e^{-\delta L_{i, j} X_{i, j} I\left(L_{i, j}>t\right)}} \mid L_{i, 1}=v_{1}, \ldots, L_{i, k}=v_{k}\right] d W_{1}\left(v_{1}\right) \cdots d W_{k}\left(v_{k}\right)
$$

and thus, from (4) and (5) with the help of (33) we get

$$
M_{\mathbf{Z}(t)}(\mathbf{s})=E\left[\prod_{i=1}^{N_{t}} M_{t-T_{i}, \mathbf{X}}^{*}\left(e^{-\delta T_{i}} \mathbf{s}\right)\right]
$$

Similar to (24), let us introduce the function associated with the report lag distribution for type- $j$ claim as

$$
\bar{\omega}_{\delta, j}(t)=\int_{t}^{\infty} e^{-\delta v} d W_{j}(v)
$$

then it is straightforward to derive the following theorem.

Theorem 3 For $n_{i}=0,1,2, \ldots$, and $\sum_{i=1}^{k} n_{i} \neq 0$, the $\mathbf{n}$-th joint moment of $k$ types of discounted total IBNR claims until $t$, satisfies the renewal equation as

$$
E\left[\prod_{j=1}^{k} Z_{j}^{n_{j}}(t)\right]=M_{\mathbf{Z}(t)}^{(\mathbf{n})}(\mathbf{0})=b_{\mathbf{n}}(t)+M_{\mathbf{Z}(\cdot)}^{(\mathbf{n})}(\mathbf{0}) * H_{\eta \delta}(t), \quad t \geq 0
$$

where $\eta=\sum_{i=1}^{k} n_{i}$. This can be evaluated recursively by using

$$
E\left[\prod_{j=1}^{k} Z_{j}^{n_{j}}(t)\right]=b_{\mathbf{n}}(t)+\int_{0}^{t} e^{-\eta \delta y} b_{\mathbf{n}}(t-y) d m(y), \quad t \geq 0
$$

where

$$
b_{\mathbf{n}}(t)=\sum_{G}\left(\begin{array}{c}
n_{1} \\
l_{1}
\end{array}\right) \cdots\left(\begin{array}{c}
n_{k} \\
l_{k}
\end{array}\right) E\left[\prod_{j \in C} X_{j}^{n_{j}-l_{j}}\right] \int_{0}^{t} e^{-\eta \delta y} E\left[\prod_{j=1}^{k} Z_{j}^{l_{j}}(t-y)\right]\left[\prod_{j \in C} \bar{\omega}_{\left(n_{j}-l_{j}\right) \delta, j}(t-y)\right] d K(y),
$$

with $G$ given in (15), $C=C_{l_{1}, \ldots, l_{k}}$ being a subset of $\{1,2, \ldots, k\}$ containing the $i$ 's such that $l_{i} \neq n_{i}$, and $\bar{\omega}_{\delta, j}(t)$ given in (34). Equivalently,

$E\left[\prod_{j=1}^{k} Z_{j}^{n_{j}}(t)\right]=\sum_{G}\left(\begin{array}{c}n_{1} \\ l_{1}\end{array}\right) \cdots\left(\begin{array}{c}n_{k} \\ l_{k}\end{array}\right) E\left[\prod_{j \in C} X_{j}^{n_{j}-l_{j}}\right] \int_{0}^{t} e^{-\eta \delta y} E\left[\prod_{j=1}^{k} Z_{j}^{l_{j}}(t-y)\right]\left[\prod_{j \in C} \bar{\omega}_{\left(n_{j}-l_{j}\right) \delta, j}(t-y)\right] d m(y)$.

Proof: The result immediately follows from Theorem 2 with the use of (33) and (34).

Then similar to Example 2, we get the mean of discounted total IBNR claims until $t$ in the following example. 
Example $3\left(k=1\right.$ and $\left.n_{1}=1\right)$ We find that (35) with (34) becomes

$$
b_{1}(t)=E\left[X_{1}\right] \int_{0}^{t} e^{-\delta y} \bar{\omega}_{\delta, 1}(t-y) d K(y)=E\left[X_{1}\right]\left[\bar{\omega}_{\delta, 1} * H_{\delta}(t)\right],
$$

and thus the mean of the discounted total IBNR claims until $t$ is obtained as

$$
E\left[Z_{1}(t)\right]=E\left[X_{1}\right]\left[\bar{\omega}_{\delta, 1} * H_{\delta} * \sum_{i=0}^{\infty} H_{\delta}^{* i}(t)\right]=E\left[X_{1}\right] \int_{0}^{t} e^{-\delta y} \bar{\omega}_{\delta, 1}(t-y) d m(y), \quad t \geq 0 .
$$

In particular, when $\delta=0$ it reduces to $E\left[Z_{1}(t)\right]=E\left[X_{1}\right] \int_{0}^{t} \bar{W}(t-y) d m(y)$.

While the asymptotic joint moment of $k$ types of discounted total reported claims was nicely obtained as in (32), in the present case of unreported claims, the joint moment for $\delta>0$ is going to be zero as $t \rightarrow \infty$. To be more precise, the joint moment of discounted aggregate unreported claims in the integral on the right-hand side of (36) is smaller than the joint moment of discounted aggregate claims until $t$ which is bounded by $(20)$, and $\bar{\omega}_{\delta, j}(y) \leq \bar{\omega}_{\delta, j}(0)=\widetilde{w}_{j}(\delta)<1$ from (34). Also, from (3), we have $\int_{0}^{\infty} e^{-\delta y} d m(y)<\infty$. Hence, by dominated convergence it follows that (36) will be zero as $t \rightarrow \infty$ due to $\lim _{t \rightarrow \infty} \bar{\omega}_{\delta, j}(t)=0$.

Moreover, it is straightforward to obtain the $\mathbf{n}$-th joint moments of $k$ types of total number of IBNR claims until time $t$ from Theorem 3. The result is given in the following corollary.

Corollary 2 For $n_{i}=0,1,2, \ldots$, and $\sum_{i=1}^{k} n_{i} \neq 0$, the $\mathbf{n}$-th joint moment of $k$ types of total number of IBNR claims until $t$, satisfies the renewal equation as

$$
E\left[\prod_{j=1}^{k} Z_{j}^{n_{j}}(t)\right]=M_{\mathbf{Z}(t)}^{(\mathbf{n})}(\mathbf{0})=\beta_{\mathbf{n}}(t)+M_{\mathbf{Z}(\cdot)}^{(\mathbf{n})}(\mathbf{0}) * H_{0}(t), \quad t \geq 0 .
$$

This also has a recursive expression given as

$$
E\left[\prod_{j=1}^{k} Z_{j}^{n_{j}}(t)\right]=\beta_{\mathbf{n}}(t)+\int_{0}^{t} \beta_{\mathbf{n}}(t-y) d m(y), \quad t \geq 0
$$

where

$$
\beta_{\mathbf{n}}(t)=\sum_{G}\left(\begin{array}{c}
n_{1} \\
l_{1}
\end{array}\right) \cdots\left(\begin{array}{c}
n_{k} \\
l_{k}
\end{array}\right) \int_{0}^{t} E\left[\prod_{j=1}^{k} Z_{j}^{l_{j}}(t-y)\right]\left[\prod_{j \in C} \bar{W}_{j}(t-y)\right] d K(y) .
$$

Alternatively, (38) can be expressed as

$$
E\left[\prod_{j=1}^{k} Z_{j}^{n_{j}}(t)\right]=\sum_{G}\left(\begin{array}{c}
n_{1} \\
l_{1}
\end{array}\right) \cdots\left(\begin{array}{c}
n_{k} \\
l_{k}
\end{array}\right) \int_{0}^{t} E\left[\prod_{j=1}^{k} Z_{j}^{l_{j}}(t-y)\right]\left[\prod_{j \in C} \bar{W}_{j}(t-y)\right] d m(y) .
$$

Proof: Setting $\delta=0$ and $X_{i, j}=1$ in (34) and (35) yields (39), and thus the result follows.

In addition, the asymptotic mean of IBNR claim can be obtained in the following corollary. 
Corollary 3 The asymptotic mean of total IBNR number of $j$-type claim in a renewal claim counting process can be obtained as

$$
E\left[Z_{j}(\infty)\right]=\frac{E\left[L_{j}\right]}{E\left[\tau_{1}\right]}
$$

Proof: Let us denote $h_{j}(t)=\int_{0}^{t} \bar{W}_{j}(t) d m(y) \equiv \bar{W}_{j} * m(t)$ where $\widetilde{h}_{j}(s)=\int_{0}^{\infty} e^{-s x} h_{j}(x) d x$. Then using (3) and $\int_{0}^{\infty} e^{-s x} \bar{W}_{j}(x) d x=\left\{1-\widetilde{w}_{j}(s)\right\} / s$, we have $\widetilde{h}_{j}(s)=\left(\frac{1-\widetilde{w}_{j}(s)}{s}\right) \frac{\widetilde{k}(s)}{1-\widetilde{k}(s)}$. Therefore, by final value theorem it follows that

$$
h_{j}(\infty)=\lim _{s \rightarrow 0} s \widetilde{h}_{j}(s)=\lim _{s \rightarrow 0} \widetilde{k}(s) \frac{1-\widetilde{w}_{j}(s)}{1-\widetilde{k}(s)}=\lim _{s \rightarrow 0} \frac{1-\widetilde{w}_{j}(s)}{1-\widetilde{k}(s)}=\lim _{s \rightarrow 0} \frac{-\widetilde{w}_{j}^{\prime}(0)}{-\widetilde{k}^{\prime}(0)},
$$

which gives rise to (41).

In particular, for the Poisson process with rate $\lambda$ (i.e. $m(t)=\lambda t),(41)$ becomes $\lambda E\left[L_{j}\right]$. Thus, for a mixed Poisson process with intensity $\Lambda$, the asymptotic mean of total IBNR number of $j$-type claim is

$$
E\left[Z_{j}(\infty)\right]=E[\Lambda] E\left[L_{1}\right]
$$

which is known in the literature (e.g. Equation (2.1.5) of Willmot (1990)).

For a general $k \geq 1$, the asymptotic joint moment of total IBNR claims can also be calculated for a mixed Poisson process with intensity $\Lambda$ as

$$
E\left[\prod_{j=1}^{k} Z_{j}^{n_{j}}(\infty)\right]=E[\Lambda] \sum_{G}\left(\begin{array}{l}
n_{1} \\
l_{1}
\end{array}\right) \cdots\left(\begin{array}{l}
n_{k} \\
l_{k}
\end{array}\right) \int_{0}^{\infty} E\left[\prod_{j=1}^{k} Z_{j}^{l_{j}}(y)\right]\left[\prod_{j \in C} \bar{W}_{j}(y)\right] d y,
$$

although it is not a recursive formula for asymptotic joint moment like (20) or (32).

\section{Numerical illustrations}

We now present numerical examples to illustrate how to calculate covariance and correlation coefficient of two types of (i) discounted aggregate claims; (ii) discounted reported claims; (iii) discounted unreported claims; and (iv) total number of IBNR claims until time $t$. The procedure requires the joint moments derived in Section 3 when $k=2$ and $n_{1}=n_{2}=1$ as well as the second moments of the univariate case of (i)-(iv) for each type of claim. Throughout this section, let $Z_{i}^{A}(t)$ be the discounted aggregate claims (type- $i$ ) until time $t$, and other superscripts " $R$ ", " $U$ ", and " $N$ " added to $Z_{i}(t)$ indicate "discounted aggregate reported claims", "discounted aggregate unreported claims", and "total number of IBNR claims" respectively. Same notation rules are applied to covariance (Cov) and correlation coefficient $(\rho)$, e.g.

$$
\operatorname{Cov}^{A}(t)=E\left[Z_{1}^{A}(t) Z_{2}^{A}(t)\right]-E\left[Z_{1}^{A}(t)\right] E\left[Z_{2}^{A}(t)\right],
$$

and

$$
\rho^{A}(t)=\frac{E\left[Z_{1}^{A}(t) Z_{2}^{A}(t)\right]-E\left[Z_{1}^{A}(t)\right] E\left[Z_{2}^{A}(t)\right]}{\sqrt{E\left[Z_{1}^{A}(t)^{2}\right]-E\left[Z_{1}^{A}(t)\right]^{2}} \sqrt{E\left[Z_{2}^{A}(t)^{2}\right]-E\left[Z_{2}^{A}(t)\right]^{2}}} .
$$

Suppose that there are two types of claim severities modeled via the bivariate gamma distribution proposed by Izawa (1953), which is constructed from gamma marginals with different scale and shape 
parameters. As discussed by Balakrishnan and Lai (2009), applications of this bivariate gamma distribution can be found in several fields such as the modeling of rainfall at two nearby rain gauges (e.g. Izawa (1965)). Then in this example, the marginal pdf of $X_{i}$ is assumed to be $\left(e^{-x / \beta_{i}} x^{\alpha-1}\right) /\left(\beta_{i}^{\alpha} \Gamma(\alpha)\right)$ with mean $\alpha \beta_{i}$, variance $\alpha \beta_{i}^{2}$ for $i=1,2$, and the covariance of $X_{1}$ and $X_{2}$ is $\alpha \beta_{1} \beta_{2} \rho$ (i.e. correlation coefficient is $\rho$ ). Also, from Izawa (1965), the joint pdf and the joint characteristic function are given by

$$
p_{X_{1}, X_{2}}\left(x_{1}, x_{2}\right)=\frac{\left(x_{1} x_{2}\right)^{(\alpha-1) / 2} \exp \left[-\frac{1}{1-\rho}\left(\frac{x_{1}}{\beta_{1}}+\frac{x_{2}}{\beta_{2}}\right)\right]}{\Gamma(\alpha)\left(\beta_{1} \beta_{2}\right)^{(\alpha+1) / 2}(1-\rho) \rho^{(\alpha-1) / 2}} I_{\alpha-1}\left(\frac{2 \sqrt{\rho}}{\sqrt{\beta_{1} \beta_{2}}(1-\rho)} \sqrt{x_{1} x_{2}}\right),
$$

where $I_{\alpha-1}(x)$ is the modified Bessel function of the first kind of order $\alpha-1$, and

$$
\phi\left(s_{1}, s_{2}\right)=\left[1+(1-\rho) \beta_{1} \beta_{2} s_{1} s_{2}+\left(\beta_{1} s_{1}+\beta_{2} s_{2}\right)\right]^{-\alpha},
$$

respectively. Note that the range of correlation coefficient in this distribution takes only non-negative value. Here we set the parameters $\alpha=2, \beta_{1}=1, \beta_{2}=5$ and $\rho=0,0.5,0.9$ (i.e. $\rho=0$ is the independent case). In other words, $E\left[X_{1}\right]=2$ and $E\left[X_{2}\right]=10$. As mentioned in Willmot (1990), the time to report/settle a claim may depend on the size of claim (e.g. large claims may be reported more quickly than small claims). Thus, the time lag distribution for the type- 1 and type- 2 claims are assumed to be exponential with mean 1 and 0.2 respectively, i.e. $W_{1}(t)=1-e^{-t}$ and $W_{2}(t)=1-e^{-5 t}$ for $t>0$. However, note that the discounted aggregate claims do not depend on the time lag distribution. Furthermore, we consider the two different claim counting processes including Poisson process with $k(t)=e^{-t}$ and Erlang $(2)$ process with $k(t)=t e^{-t}$. In addition, the discount factor $\delta$ is assumed to be $5 \%$. All the following calculations were carried out using Mathematica.

First, we calculate the expectations of four different quantities in (i)-(iv) for type-1 and type-2 claims separately (i.e. univariate case) in the Poisson process. A summary of results is given in Table 1. All values are increasing in $t$ except for the discounted aggregate unreported claims. The asymptotic expectations for each quantity of interest can be also confirmed numerically. For example, the average total number of IBNR claim as $t \rightarrow \infty$ is essentially the mean of time lag distribution (due to $E\left[\tau_{1}\right]=1$ in the Poisson example), and thus the means are obtained as $E\left[L_{1}\right]=1$ for type-1 claim and $E\left[L_{2}\right]=0.2$ for type-2 claim respectively. In particular, as discussed in Section 3.2, the asymptotic moment of discounted aggregate unreported claims is going to be zero as $t \rightarrow \infty$. Since the mean of type- 2 claim is larger than the the mean of type- 1 claim, and the reporting delay for type- 2 claim is shorter than type-1 claim, all expectations of discounted aggregate claims and reported claims are much larger than those of type-1 claim. On the contrary, for the unreported claims and its total number, expectations for type-2 claim are smaller.

\begin{tabular}{|r|rr|rr|rr|rr|}
\hline$t$ & $E\left[Z_{1}^{A}(t)\right]$ & $E\left[Z_{2}^{A}(t)\right]$ & $E\left[Z_{1}^{R}(t)\right]$ & $E\left[Z_{2}^{R}(t)\right]$ & $E\left[Z_{1}^{U}(t)\right]$ & $E\left[Z_{2}^{U}(t)\right]$ & $E\left[Z_{1}^{N}(t)\right]$ & $E\left[Z_{2}^{N}(t)\right]$ \\
\hline 1 & 1.9508 & 9.7541 & 0.7126 & 7.7866 & 1.1453 & 1.8709 & 0.6321 & 0.1987 \\
5 & 8.8480 & 44.2398 & 6.9532 & 42.2596 & 1.4734 & 1.5422 & 0.9933 & 0.2000 \\
10 & 15.7388 & 78.6939 & 13.8341 & 76.7137 & 1.1552 & 1.2011 & 0.9999 & 0.2000 \\
100 & 39.7305 & 198.6520 & 37.8257 & 196.6720 & 0.0128 & 0.0133 & 1.0000 & 0.2000 \\
1000 & 40.0000 & 200.0000 & 38.0952 & 198.0200 & 0.0000 & 0.0000 & 1.0000 & 0.2000 \\
$\infty$ & 40.0000 & 200.0000 & 38.0952 & 198.0200 & 0.0000 & 0.0000 & 1.0000 & 0.2000 \\
\hline
\end{tabular}

Table 1: Expectations of (i)-(iv) for each type of claim in the Poisson process

Next, Tables 2-4 presents covariances and correlations between type-1 and type-2's (i)-(iv) by implementing the results of Theorems 1-3 and Corollary 2 with different correlation coefficient of $X_{1}$ and $X_{2}$, which include the cases of $\rho=0,0.5$ and 0.9 respectively. Note that $\operatorname{Cov}^{N}$ and $\rho^{N}$ are 
excluded in Tables 3-4 since these quantities are not affected by the (dependency between) the two claim sizes. As was evident in Tables 2-4, as $t$ increases, while $\operatorname{Cov}^{A}(t)$ and $\operatorname{Cov}^{R}(t)$ exhibit a similar increasing trend, $\mathrm{Cov}^{U}(t)$ decreases. Moreover, due to the concurrent impact from the same claim incurral process on the occurrence of type- 1 and type- 2 claims, positive dependencies are observed through correlation coefficients of all quantities (i)-(iv) for both types. In particular, Tables 3-4 enable us to assert the impact of correlation between different types of claim severities on the relation between their discounted aggregate quantities listed in (i)-(iii). As $\rho$ between $X_{1}$ and $X_{2}$ gets larger, correlations between $Z_{i}^{A}(t)$ 's, $Z_{R}^{A}(t)$ 's, and $Z_{i}^{U}(t)$ 's (for $i=1,2$ ) also become stronger.

\begin{tabular}{|r|rr|rr|rr|rr|}
\hline$t$ & $\operatorname{Cov}^{A}(t)$ & $\rho^{A}(t)$ & $\operatorname{Cov}^{R}(t)$ & $\rho^{R}(t)$ & $\operatorname{Cov}^{U}(t)$ & $\rho^{U}(t)$ & $\operatorname{Cov}^{N}(t)$ & $\rho^{N}(t)$ \\
\hline 1 & 19.0325 & 0.6667 & 6.34517 & 0.4139 & 2.8370 & 0.3124 & 0.1663 & 0.4692 \\
5 & 78.6939 & 0.6667 & 61.8637 & 0.6128 & 1.9064 & 0.2490 & 0.1667 & 0.3739 \\
10 & 126.4241 & 0.6667 & 111.6640 & 0.6465 & 1.1563 & 0.2482 & 0.1667 & 0.3727 \\
100 & 199.9909 & 0.6667 & 188.5808 & 0.6659 & 0.0001 & 0.2482 & 0.1667 & 0.3727 \\
500 & 199.9999 & 0.6667 & 188.5903 & 0.6659 & 0.0000 & 0.2482 & 0.1667 & 0.3727 \\
1000 & 200.0000 & 0.6667 & 188.5903 & 0.6659 & 0.0000 & 0.2482 & 0.1667 & 0.3727 \\
\hline
\end{tabular}

Table 2: Covariances and Correlation coefficients of (i)-(iv) in the Poisson process $(\rho=0)$

\begin{tabular}{|r|rr|rr|rr|}
\hline$t$ & $\operatorname{Cov}^{A}(t)$ & $\rho^{A}(t)$ & $\operatorname{Cov}^{R}(t)$ & $\rho^{R}(t)$ & $\operatorname{Cov}^{U}(t)$ & $\rho^{U}(t)$ \\
\hline 1 & 23.7906 & 0.8333 & 7.9315 & 0.5173 & 3.5463 & 0.3905 \\
5 & 98.3673 & 0.8333 & 77.3296 & 0.7659 & 2.3830 & 0.3112 \\
10 & 158.0301 & 0.8333 & 139.5801 & 0.8081 & 1.4454 & 0.3102 \\
100 & 249.9887 & 0.8333 & 235.7260 & 0.8323 & 0.0002 & 0.3102 \\
500 & 249.9999 & 0.8333 & 235.7379 & 0.8323 & 0.0000 & 0.3102 \\
1000 & 250.0000 & 0.8333 & 235.7379 & 0.8323 & 0.0000 & 0.3102 \\
\hline
\end{tabular}

Table 3: Covariances and Correlation coefficients of (i)-(iv) in the Poisson process $(\rho=0.5)$

\begin{tabular}{|r|rr|rr|rr|}
\hline$t$ & $\operatorname{Cov}^{A}(t)$ & $\rho^{A}(t)$ & $\operatorname{Cov}^{R}(t)$ & $\rho^{R}(t)$ & $\operatorname{Cov}^{U}(t)$ & $\rho^{U}(t)$ \\
\hline 1 & 27.5971 & 0.9667 & 9.2005 & 0.6001 & 4.1137 & 0.4530 \\
5 & 114.1061 & 0.9667 & 89.7023 & 0.8885 & 2.7643 & 0.3610 \\
10 & 183.3150 & 0.9667 & 161.9129 & 0.9374 & 1.6767 & 0.3598 \\
100 & 289.9868 & 0.9667 & 273.4422 & 0.9655 & 0.0002 & 0.3598 \\
500 & 289.9999 & 0.9667 & 273.4559 & 0.9655 & 0.0000 & 0.3598 \\
1000 & 290.0000 & 0.9667 & 273.4559 & 0.9655 & 0.0000 & 0.3598 \\
\hline
\end{tabular}

Table 4: Covariances and Correlation coefficients of (i)-(iv) in the Poisson process $(\rho=0.9)$

Furthermore, those quantities in Tables 1-4 are calculated again under the Erlang(2) claim incurral process. Similar patterns to Tables 1-4 are observed in this process. Note that the absolute values in Tables 5-8 are smaller than counterparts of Poisson case, whereas the variabilities of the values of $E\left[Z_{i}(t)\right], \operatorname{Cov}(t)$, and $\rho(t)$ from time 0 to 1000 are larger under the Erlang $(2)$ process. It can be explained by the fact that the mean and the variance of $\operatorname{Erlang}(2)$ interclaim time distribution are both 2 which are larger than those of Poisson risk process (i.e. $E\left[\tau_{1}\right]=\operatorname{Var}\left(\tau_{1}\right)=1$ in the exponential interclaim time distribution). In addition, the asymptotic means of total number of IBNR claim, i.e. $E\left[Z_{1}^{N}(\infty)\right]$ and $E\left[Z_{2}^{N}(\infty)\right]$ are reduced by $50 \%$ compared to the values in the Poisson process as the 
mean of interclaim distribution is changed from 1 to 2 , which confirms the result in Corollary 3 .

\begin{tabular}{|r|rr|rr|rr|rr|}
\hline$t$ & $E\left[Z_{1}^{A}(t)\right]$ & $E\left[Z_{2}^{A}(t)\right]$ & $E\left[Z_{1}^{R}(t)\right]$ & $E\left[Z_{2}^{R}(t)\right]$ & $E\left[Z_{1}^{U}(t)\right]$ & $E\left[Z_{2}^{U}(t)\right]$ & $E\left[Z_{1}^{N}(t)\right]$ & $E\left[Z_{2}^{N}(t)\right]$ \\
\hline 1 & 0.5504 & 2.7520 & 0.1622 & 1.9912 & 0.3620 & 0.7336 & 0.1998 & 0.0779 \\
5 & 3.9362 & 19.6810 & 3.0170 & 18.7151 & 0.7318 & 0.7710 & 0.4933 & 0.0999 \\
10 & 7.3816 & 36.9079 & 6.4525 & 35.9420 & 0.5776 & 0.6005 & 0.4999 & 0.0999 \\
100 & 19.3774 & 96.8872 & 18.4483 & 95.9212 & 0.0006 & 0.0007 & 0.5000 & 0.1000 \\
1000 & 19.5122 & 97.5610 & 18.5830 & 96.5950 & 0.0000 & 0.0000 & 0.5000 & 0.1000 \\
$\infty$ & 19.5122 & 97.5610 & 18.5830 & 96.5950 & 0.0000 & 0.0000 & 0.5000 & 0.1000 \\
\hline
\end{tabular}

Table 5: Expectations of (i)-(iv) for each type of claim in the Erlang(2) process

\begin{tabular}{|r|rr|rr|rr|rr|}
\hline$t$ & $\operatorname{Cov}^{A}(t)$ & $\rho^{A}(t)$ & $\operatorname{Cov}^{R}(t)$ & $\rho^{R}(t)$ & $\operatorname{Cov}^{U}(t)$ & $\rho^{U}(t)$ & $\operatorname{Cov}^{N}(t)$ & $\rho^{N}(t)$ \\
\hline 1 & 4.5855 & 0.6321 & 1.2074 & 0.3442 & 0.9811 & 0.3245 & 0.0575 & 0.5018 \\
5 & 20.3631 & 0.5408 & 15.6816 & 0.4790 & 0.7263 & 0.2070 & 0.0635 & 0.3254 \\
10 & 31.9913 & 0.5226 & 28.0290 & 0.4992 & 0.4405 & 0.2055 & 0.0635 & 0.3228 \\
100 & 49.9114 & 0.5118 & 47.0638 & 0.5109 & 0.0001 & 0.2055 & 0.0635 & 0.3228 \\
500 & 49.9136 & 0.5118 & 47.0661 & 0.5109 & 0.0000 & 0.2055 & 0.0635 & 0.3228 \\
1000 & 49.9136 & 0.5118 & 47.0661 & 0.5109 & 0.0000 & 0.2055 & 0.0635 & 0.3228 \\
\hline
\end{tabular}

Table 6: Covariances and Correlation coefficients of (i)-(iv) in the $\operatorname{Erlang}(2)$ process $(\rho=0)$

\begin{tabular}{|r|rr|rr|rr|}
\hline$t$ & $\operatorname{Cov}^{A}(t)$ & $\rho^{A}(t)$ & $\operatorname{Cov}^{R}(t)$ & $\rho^{R}(t)$ & $\operatorname{Cov}^{U}(t)$ & $\rho^{U}(t)$ \\
\hline 1 & 5.9199 & 0.8161 & 1.5481 & 0.4413 & 1.2649 & 0.4183 \\
5 & 29.0094 & 0.7704 & 22.3038 & 0.6813 & 0.9646 & 0.2749 \\
10 & 46.6038 & 0.7613 & 40.8646 & 0.7278 & 0.5850 & 0.2729 \\
100 & 73.7198 & 0.7559 & 69.5138 & 0.7546 & 0.0001 & 0.2729 \\
500 & 73.7231 & 0.7559 & 69.5173 & 0.7546 & 0.0000 & 0.2729 \\
1000 & 73.7231 & 0.7559 & 69.5173 & 0.7546 & 0.0000 & 0.2729 \\
\hline
\end{tabular}

Table 7: Covariances and Correlation coefficients of (i)-(iv) in the $\operatorname{Erlang}(2)$ process $(\rho=0.5)$

\section{Concluding remarks}

The proposed model in this paper has the merit of providing a unified framework in studying the joint moments of the discounted total multivariate claims in the presence of reporting/payment delay. We should point out that most studies in the literature related to the discounted aggregate (reported/unreported) claims (i.e. without delay or with delay in reporting) are done separately under various claim counting processes such as renewal processes and a (non-homogeneous or mixed) Poisson processes with univariate claim severities.

As shown in this paper, the extension to the case of aggregate multivariate claim severities involving the delay component in terms of reporting/payment is performed by using the renewal arguments. Utilizing the regenerative property at claim instants assumed in the renewal claim counting process, the joint moments of the aggregate discounted claims with univariate claim severity were studied by Léveillé and Garrido (2001a,b) and Woo and Cheung (2013). To find the joint moments of $\left\{Z_{j}(t)\right\}_{j \in \mathrm{R}^{k}}$, the result from combinatorics of partial derivatives by Hardy (2006) is adopted. It is of theoretical and 


\begin{tabular}{|r|rr|rr|rr|}
\hline$t$ & $\operatorname{Cov}^{A}(t)$ & $\rho^{A}(t)$ & $\operatorname{Cov}^{R}(t)$ & $\rho^{R}(t)$ & $\operatorname{Cov}^{U}(t)$ & $\rho^{U}(t)$ \\
\hline 1 & 6.9874 & 0.9632 & 1.8206 & 0.5190 & 1.4919 & 0.4934 \\
5 & 35.9265 & 0.9541 & 27.6015 & 0.8431 & 1.1552 & 0.3293 \\
10 & 58.2938 & 0.9523 & 51.1330 & 0.9107 & 0.7007 & 0.3268 \\
100 & 92.7665 & 0.9512 & 87.4738 & 0.9495 & 0.0001 & 0.3268 \\
500 & 92.7707 & 0.9512 & 87.4783 & 0.9495 & 0.0000 & 0.3268 \\
1000 & 92.7707 & 0.9512 & 87.4783 & 0.9495 & 0.0000 & 0.3268 \\
\hline
\end{tabular}

Table 8: Covariances and Correlation coefficients of (i)-(iv) in the $\operatorname{Erlang}(2)$ process $(\rho=0.9)$

practical interest to investigate the risk aggregation of the multiline insurance, especially when claim severities are dependent on their incurral time. Moreover, we remark that the expressions for the joint moment of the discounted multivariate claim obtained in Section 3 are recursively evaluated with the aid of the renewal function $m(t)$ and the mixed moment of $\left(X_{1}, X_{2}, \ldots, X_{k}\right)$ of order $\left(n_{1}, n_{2}, \ldots, n_{k}\right)$. The former quantity is simple and well known in some processes but is not explicitly available in some other processes. However, bounds and asymptotic properties for $m(t)$ are broadly studied in the area of renewal theory. The latter quantity is also explicitly given for many multivariate distributions, e.g. Moran and Downton's multivariate exponential (Equation (47.119) of Kotz et al. (2000)). Finally, as demonstrated by the previous examples in Section 4, we note that unified approach given in Section 3 is easily implemented to study the impact of dependency between different types of claims on the relation of their discounted (reported/unreported) aggregate amounts as well as to obtain the asymptotic joint moments under the different claim incurral processes.

Acknowledgements. The author is grateful to the anonymous referee for several helpful comments and suggestions, which have lead to improvements in the paper. This work has been supported by the CAE 2013 research grant from the Society of Actuaries. Any opinions, findings, and conclusions or recommendations expressed in this material are those of the author and do not necessarily reflect the views of the SOA.

\section{References}

[1] Alink, S., LÖWe, M., And Wüthrich, M.V. 2005. Analysis of the expected shortfall of aggregate dependent risks. ASTIN Bulletin 35(1): 25-43.

[2] Asimit, A.V., Furman, E., and Vernic, R. 2010. On a multivariate Pareto distribution. Insurance: Mathematics and Economics 46(2): 308-316.

[3] Assaf, D., Landberg, N., Savits, T., and Shaked, M. 1984. Multivariate phase-type distributions. Operations Research 32(3): 688-702.

[4] Balakrishnan, N. And Lai, C.-D. 2009. Continuous Bivariate Distributios. 2nd Edition. Springer.

[5] CAI, J. AND LI, H. 2005a. Multivariate risk model of phase type. Insurance: Mathematics and Economics 36(2): 137-152.

[6] CAI, J. AND Li, H. 2005b. Conditional tail expectations for multivariate phase-type distributions. Journal of Applied Probability 42(3): 810-825. 
[7] Chiragiev, A. And Landsman, Z. 2007. Multivariate Pareto portfolios: TCE-based capital allocation and divided differences. Scandinavian Actuarial Journal 4: 261-280.

[8] Cossette, H., Côté, M.-P., and Marceau, É., Moutanabbir, K. 2013. Multivariate distribution defined with Farlie-Gumbel-Morgenstern copula and mixed Erlang marginals: Aggregation and capital allocation. Insurance: Mathematics and Economics 52(3): 560-572.

[9] Furman, E., And Landsman, Z. 2010. Multivariate Tweedie distributions and some related capital-at-risk analyses. Insurance: Mathematics and Economics 46(2): 351-361.

[10] Grandell, J. 1997. Mixed Poisson Processes. Chapman \& Hall: London.

[11] Guo, L., Landriault, D., and Willmot, G.E. 2013. On the analysis of a class of loss models incorporating time dependence. European Actuarial Journal 3(1): 273-294.

[12] Hardy, M. 2006. Combinatorics of partial derivatives. The Electronic Journal of Combinatorics 13(1): Research Paper R1.

[13] Izawa, T. 1953. The bivariate gamma distribution. Climate and Statistics 4: 9-15. (in Japanese)

[14] Izawa, T. 1965. Two or multidimensional gamma-type distribution and its application to rainfall data. Papers in Meteorology and Geophysics 15: 167-200.

[15] Joe, H. 1997. Multivariate Models and Dependence Concepts. Chapman \& Hall: London.

[16] Karlsson, J.-E. 1974. A stochastic model for time lag in reporting of claims. Journal of Applied Probability 11: 382-387.

[17] Kotz, S., Balakrishnan, N. And Johnson, N.L. 2000. Continuous Multivariate Distributions: Volume 1, Models and Applications. 2nd Edition. Wiley.

[18] Kulkarni, V.G. 1989. A new class of multivariate phase type distributions. Operations Research 37(1): 151-158.

[19] Landriault, D., Willmot, G.E., And Xu, D. 2014. On the analysis of time dependent claims in a class of birth process claim count models. Insurance: Mathematics and Economics 58: 168173.

[20] LeE, S.C.K. And Lin, X.S. 2012. Modeling dependent risks with multivariate Erlang mixtures. ASTIN Bulletin 42(1): 153-180.

[21] LÉveillé, G. ANd AdÉKAmbi, F. 2011. Covariance of discounted compound renewal sums with a stochastic interest rate. Scandinavian Actuarial Journal 2: 138-153.

[22] LÉveillé, G. AND Garrido, J. 2001a. Moments of compound renewal sums with discounted claims. Insurance: Mathematics and Economics 28(2): 217-231.

[23] LÉveillé, G. And Garrido, J. 2001b. Recursive moments of compound renewal sums with discounted claims. Scandinavian Actuarial Journal 2: 98-110.

[24] Léveillé, G., Garrido, J. and Wang, Y.F. 2010. Moment generating functions of compound renewal sums with discounted claims. Scandinavian Actuarial Journal 3: 165-184. 
[25] LÉveillé, G. And HAMEl, E. 2013. A compound renewal model for medical malpractice insurance. European Actuarial Journal 3: 471-490.

[26] PAnjer, H.H. 2002. Measurement of risk, solvency requirements, and allocation of capital within financial conglomerates. Institute of Insurance and Pension Research, University of Waterloo Research Report: 1-15.

[27] Tiums, H.C. 2003. A First Course in Stochastic Models. Wiley.

[28] Willmot, G.E. 1989. The total claims distribution under inflationary conditions. Scandinavian Actuarial Journal 1: 1-12.

[29] Willmot, G.E. 1990. A queueing theoretic approach to the analysis of the claims payment process. Transaction of Society of Actuaries 42: 447-497.

[30] Willmot, G.E. And Woo, J.-K. 2015. On some properties of a class of multivariate Erlang mixtures with insurance applications. ASTIN Bulletin 45(1): 151-173.

[31] Woo, J.-K. And Cheung, E.C.K. 2013. A note on discounted compound renewal sums under dependency. Insurance: Mathematics and Economics 52(2): 170-179. 